\title{
Heterodyne Efficiency for a Coherent Laser Radar with Diffuse or Aerosol Targets
}

\author{
R. G. Frehlich \\ Cooperative Institute for Research in the Environmental Sciences (CIRES) \\ University of Colorado, Boulder, CO 80309
}

$\begin{array}{lll}n & & \\ 5 & n & 0 \\ i & 0 & 0 \\ 1 & 0 & 0 \\ 5 & u & 0 \\ 2 & 5 & 0\end{array}$

\section{Abstract}

The performance of a Coherent Laser Radar is determined by the statistics of the coherent Doppler signal. The heterodyne efficiency is a excellent indication of performance because it is an absolute measure of beam alignment and is independent of the transmitter power, the target backscatter coefficient, the atmospheric attenuation, and the detector quantum efficiency and gain. The theoretical calculation of heterodyne efficiency for an optimal monostatic lidar with a circular aperture and Gaussian transmit laser is presented including beam misalignment in the far-field and near-field regimes. The statistical behavior of estimates of the heterodyne efficiency using a calibration hard target are considered. For space based applications, a biased estimate of heterodyne efficiency is proposed that removes the variability due to the random surface return but retains the sensitivity to misalignment. Physical insight is provided by simulation of the fields on the detector surface. The required detector calibration is also discussed.

Key words: Lidar, Laser Radar, Remote Sensing.

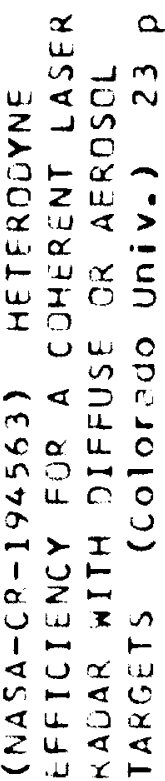

\section{Introduction}

An important measure of the performance of coherent laser radar (CLR) is the Signal-toNoise Ratio (SNR) defined by [1]

$S N R(t)=\frac{\left\langle i_{S}^{2}(t)\right\rangle}{\left\langle i_{N}^{2}\right\rangle}$

where (\rangle denotes ensemble average, $\left\langle i_{S}^{2}(t)\right\rangle\left[A^{2}\right]$ is the average coherent power at time $t[s]$, and $\left\langle i_{N}^{2}(t)\right\rangle\left[A^{2}\right]$ is the average noise power. For an infinite diffuse target at range $R[m]$ with uniform backscatter coefficient $\rho$, detector noise dominated by Local Oscillator (LO) shot noise, a detector with uniform quantum efficiency $\eta_{Q}$ that collects all the energy of the LO beam, the CLR SNR is given by $[1,2]$

$S N R(R)=\frac{\eta_{Q} P_{L} \rho K^{2}}{h \nu B} C(R)$

where $P_{L}[W]$ is the transmitted laser power, $h[J s]$ is Plancks constant, $\nu\left[s^{-1}\right]$ is the frequency of the laser, $B\left[s^{-1}\right]$ is the signal bandwidth of the detector, $K$ is the one-way atmospheric 
attenuation, and $C(R)$ is the coherent responsivity. For an aerosol target, similar expressions are obtained [Ref. [ 1], Eqs. (89), (92)]. When the effects of atmospheric refractive turbulence are negligible

$C(R)=A_{R} T_{T} \eta_{H}(R) / R^{2}$

where $A_{R}\left[\mathrm{~m}^{2}\right]$ is the area of the telescope aperture, $T_{T}$ is the fraction of the laser power transmitted through the telescope aperture, and $\eta_{H}(R)$ is the heterodyne efficiency. Heterodyne efficiency is unity when the backscattered field is proportional to the LO field on the detector surface. Heterodyne efficiency is a useful measure of performance for a CLR because it is independent of the transmitter power, the backscatter coefficient, the detector quantum efficiency, and the atmospheric attenuation. The heterodyne efficiency can also be estimated from the detector signal [1], i.e.,

$\eta_{H}(t)=\frac{\left\langle i_{S}^{2}(t)\right\rangle}{2\left\langle I_{D C}\right\rangle\left\langle I_{S}(t)\right\rangle}$

where $I_{D C}[A]$ is the direct current (DC) due to the LO, $i_{S}(t)[A]$ is the intermediate frequency (IF) signal current or coherent signal, and $I_{S}(t)[A]$ is the direct detection signal current due to the backscattered field [Note the typo in Ref. [1], Eq. (15)]. For diffuse and aerosol targets, the backscattered field at the receiver is spatially random and described by "specklen statistics, i.e., the complex field has a zero-mean joint Gaussian probability density function (PDF). The statistical behavior of estimates for the heterodyne efficiency will be investigated with theoretical calculations and numerical simulations.

\section{Theory}

The analysis of heterodyne efficiency is presented using the normalized direct detection power

$d=I_{S} /\left\langle I_{S}\right\rangle$

and the normalized coherent detection power

$c=\frac{i_{S}^{2}}{2\left\langle I_{D C}\right\rangle\left\langle I_{S}\right\rangle}$

Then $\langle d\rangle=1$ and $\langle c\rangle=\eta_{H}$.

The heterodyne efficiency depends on the CLR design, which can be represented by [ 1] the transmitter aperture function $W_{T}(\overrightarrow{\mathrm{u}})$, the normalized transmitter laser field incident on the transmitter aperture $e_{L}(\overrightarrow{\mathbf{u}}, 0)\left[\mathrm{m}^{-1}\right]$, the receiver aperture function $W_{R}(\overrightarrow{\mathbf{v}})$, and the normalized Local Oscillator (LO) field at the receiver aperture $e_{L O}(\vec{v}, 0)\left[m^{-1}\right]$. Here, $\vec{u}[m]$ and $\vec{v}[m]$ denote the two-dimensional transverse vector for the transmitter aperture and receiver aperture, respectively.

We will consider a monostatic CLR $\left[W_{T}(\overrightarrow{\mathbf{u}})=W_{R}(\overrightarrow{\mathbf{u}})=W(\overrightarrow{\mathbf{u}})\right]$ with a circular telescope aperture of radius $a[m]$, i.e., 


$$
\begin{aligned}
W(\overrightarrow{\mathrm{u}}) & =1 \\
& =\dot{0}
\end{aligned} \quad \begin{array}{ll}
|u| \leq a \\
\end{array}
$$

and

$A_{R}=\int_{-\infty}^{\infty}|W(\overrightarrow{\mathbf{v}})|^{2} d \overrightarrow{\mathrm{v}}=\pi a^{2}=\pi D^{2} / 4$

where $D[m]$ is the diameter of the aperture.

The heterodyne efficiency can be written as [1]

$\eta_{H}(R)=\frac{R^{2} \lambda^{2}}{A_{R} T_{T}} \int_{-\infty}^{\infty} j_{T}(\overrightarrow{\mathbf{p}}, R) j_{B P L O}(\overrightarrow{\mathbf{p}}, R) d \overrightarrow{\mathbf{p}}$

where

$j_{T}(\overrightarrow{\mathbf{p}}, R)=\left|e_{T}(\overrightarrow{\mathbf{p}}, R)\right|^{2}$

$j_{B P L O}(\dot{\overrightarrow{\mathbf{p}}}, R)=\left|e_{B P L O}(\overrightarrow{\mathbf{p}}, R)\right|^{2}$

are the irradiance $\left[\mathrm{m}^{-2}\right]$ of the normalized transmitter and Back Propagated Local Oscillator (BPLO) field, respectively. Here,

$e_{T}(\overrightarrow{\mathbf{p}}, R)=\int_{-\infty}^{\infty} e_{T}(\overrightarrow{\mathbf{u}}, 0) G(\overrightarrow{\mathbf{p}} ; \overrightarrow{\mathbf{u}}, R) d \overrightarrow{\mathbf{u}}$

is the normalized transmitter field at the target coordinate $(\overrightarrow{\mathbf{p}}, R)[\mathrm{m}]$,

$e_{T}(\overrightarrow{\mathbf{u}}, 0)=e_{L}(\overrightarrow{\mathbf{u}}, 0) W(\overrightarrow{\mathbf{u}})$

is the normalized transmitter field at the telescope aperture,

$e_{B P L O}(\overrightarrow{\mathbf{p}}, R)=\int_{-\infty}^{\infty} e_{B P L O}(\overrightarrow{\mathbf{v}}, 0) G(\overrightarrow{\mathbf{p}} ; \overrightarrow{\mathbf{v}}, R) d \overrightarrow{\mathbf{v}}$

is the normalized BPLO field at the target,

$e_{B P L O}(\overrightarrow{\mathbf{v}}, 0)=e_{L}^{*}(\overrightarrow{\mathbf{v}}, 0) W(\overrightarrow{\mathbf{v}})$

is the normalized BPLO field at the receiver aperture,

$G(\overrightarrow{\mathbf{p}} ; \overrightarrow{\mathbf{u}}, R)=\frac{k}{2 \pi i R} \exp \left[\frac{i k}{2 R}(\overrightarrow{\mathbf{p}}-\overrightarrow{\mathbf{u}})^{2}\right]$

is the free space Green's function $\left[\mathrm{m}^{-2}\right]$ for propagating the scalar fields from the transmitterreceiver plane to the target plane under the Fresnel approximation where $k\left[m^{-1}\right]=2 \pi / \lambda$ is the wavenumber of the transmitter field, and $\lambda[m]$ is the wavelength of the field.

The integrated normalized irradiance

$\int_{-\infty}^{\infty} j_{T}(\overrightarrow{\mathbf{p}}, R) d \overrightarrow{\mathbf{p}}=T_{T}$ 
is equal to the power transmittance $T_{T}$ of the laser through the transmitter-receiver aperture. Similarly,

$\int_{-\infty}^{\infty} j_{B P L O}(\overrightarrow{\mathbf{p}}, R) d \overrightarrow{\mathbf{p}}=T_{R}$

is equal to the power transmittance $T_{R}$ of the BPLO field through the transmitter-receiver aperture.

A Gaussian spatial distribution is the most common laser transmitter field, i.e.,

$e_{L}(\overrightarrow{\mathbf{u}}, 0)=\left(2 \pi \sigma_{L}^{2}\right)^{-1 / 2} \exp \left(-\frac{u^{2}}{2 \sigma_{L}^{2}}-\frac{i k u^{2}}{2 F_{L}^{2}}\right)$

where $\sigma_{L}[\mathrm{~m}]$ is the $1 / \mathrm{e}$ intensity radius of the beam at the transmitter aperture, and $F_{L}[\mathrm{~m}]$ is the radius of curvature of the phase front. The irradiance at the target is given by [substitute Eqs. (13) and (19) into (12), convert to polar coordinates and integrate over angle]

$j_{T}(\overrightarrow{\mathbf{p}}, R)=\frac{\delta_{R F}^{2}}{\pi \sigma_{L}^{2}}\left|Y\left(\Omega_{T}^{2}, k p a / R\right)\right|^{2}$

where

$\Omega_{T}^{2}=\delta_{T}^{2}-i \delta_{R F}^{2}\left(1-R / F_{L}\right)$

$\delta_{T}=a / \sigma_{L}, \delta_{R F}=a / R_{F}, R_{F}=(R / k)^{1 / 2}$

$Y(\Omega, q)=\int_{0}^{1} \exp \left(-x^{2} \Omega^{2} / 2\right) J_{0}(q x) x d x$

An efficient series expansion for $Y(\Omega, q)$ is [ 3]

$$
\begin{array}{rlrl}
Y(\Omega, q) & =\Omega^{-2}\left[\exp \left(-\frac{q^{2}}{2 \Omega^{2}}\right)-\exp \left(-\frac{\Omega^{2}}{2}\right) \sum_{n=0}^{\infty}\left(\frac{-q}{\Omega^{2}}\right)^{n} J_{n}(q)\right] & q<\left|\Omega^{2}\right| \\
& =\Omega^{-2} \exp \left(-\Omega^{2} / 2\right) \sum_{n=1}^{\infty}\left(\frac{\Omega^{2}}{q}\right)^{n} J_{n}(q) & & q>\left|\Omega^{2}\right|
\end{array}
$$

The far-field result is produced when $\Omega_{T}^{2}=\delta_{T}^{2}$, which occurs when $R=F_{L}$ (focused condition). For a collimated beam $\left(F_{L}=\infty\right)$, the far-field condition is $\delta_{T} \gg \delta_{R F}$ or $R_{F} \gg \sigma_{L}$.

A Gaussian field focused on the detector is a common choice for the LO field. The receiver-plane representation of the LO field is

$e_{L O}(\overrightarrow{\mathbf{v}}, 0)=\left(2 \pi \sigma_{L O}^{2}\right)^{-1 / 2} \exp \left(-\frac{v^{2}}{2 \sigma_{L O}^{2}}+\frac{i k v^{2}}{2 F_{L O}^{2}}\right)$

and

$j_{B P L O}(\overrightarrow{\mathbf{p}}, R)=\frac{\delta_{R F}^{2}}{\pi \sigma_{L O}^{2}}\left|Y\left(\Omega_{R}^{2}, k p a / R\right)\right|^{2}$ 
where:

$\Omega_{R}^{2}=\delta_{R}^{2}-i \delta_{R F}^{2}\left(1-R / F_{L O}\right), \quad \delta_{R}=a / \sigma_{L O}$

In practice, it is difficult to perfectly align the LO field with the backscattered field. The misalignment angle $\Delta \theta[\mathrm{rad}]$ produces an offset $\Delta p=R \Delta \theta[\mathrm{m}]$ between the two irradiance distributions in the target plane. (The effects of refractive turbulence on beam misalignment for Gaussian beams and a Gaussian aperture were investigated by Frehlich [4]). For this CLR, the heterodyne efficiency including misalignment is given by

$\eta_{H}(R)=\frac{4 \delta_{T}^{2} \delta_{R}^{2}}{\pi T_{T}} Q\left(\Omega_{T}, \Omega_{R}, \Delta q\right)$

where

$Q\left(\Omega_{T}, \Omega_{R}, \Delta q\right)=\int_{-\infty}^{\infty}\left|Y\left(\Omega_{T}, \overrightarrow{\mathbf{q}}\right)\right|^{2}\left|Y\left(\Omega_{R}, \overrightarrow{\mathbf{q}}-\Delta \overrightarrow{\mathbf{q}}\right)\right|^{2} d \overrightarrow{\mathbf{q}}$

is the overlap integral of the normalized transmitter irradiance $\left|Y\left(\Omega_{T}, \overrightarrow{\mathbf{q}}\right)\right|^{2}$ and BPLO irradiance $\left|Y\left(\Omega_{R}, \overrightarrow{\mathbf{q}}-\Delta \overrightarrow{\mathrm{q}}\right)\right|^{2}$ and

$\Delta q=\pi D \Delta \theta / \lambda$

is the normalized angle-misalignment.

Wang [5] calculated the optimal parameters for a Gaussian transmit beam and a Gaussian LO beam focused on the detector for the far-field condition $\left[\delta_{T}=\delta_{R}=1.7633\right.$, $\left.\eta_{H}=0.41992, T_{T}=T_{R}=1-\exp \left(-\delta_{T}^{2}\right)=0.95536\right]$. Rye [6] proposed an optimal design where the BPLO beam does not have any truncation by the telescope aperture, i.e., $T_{R}=1$. For this design

$\eta_{H}(R)=\frac{4 \delta_{T}^{2} \delta_{R}^{2}}{\pi T_{T}\left[1-\exp \left(-\delta_{R}^{2}\right)\right]} Q\left(\Omega_{T}, \Omega_{R}, \Delta q\right)$

and for a Gaussian LO beam, the optimal parameters are $\delta_{T}=1.7367, T_{T}=1-\exp \left(-\delta_{T}^{2}\right)=$ $0.95101, \delta_{R}=1.1931, \eta_{H}=0.460958$. The difference between these two designs is discussed by Rye and Frehlich [ 7].

For a collimated lidar, the near field behavior of heterodyne efficiency can be approximated by

$\eta_{H N F}(R)=\frac{R^{2} \lambda^{2}}{A_{R} T_{T}} \int_{-\infty}^{\infty} j_{T}(\overrightarrow{\mathbf{p}}, 0) j_{B P L O}(\overrightarrow{\mathbf{p}}, 0) d \overrightarrow{\mathbf{p}}$

For the collimated Gaussian lidar with the Rye design [see Eqs. (11), (13), (15), (16), (19), (25), (32) and normalize the BPLO field to produce $T_{R}=1$ ]

$\eta_{H N F}(R)=\frac{4 \delta_{T}^{2} \delta_{R}^{2}\left[1-\exp \left(-\delta_{T}^{2}-\delta_{R}^{2}\right)\right]}{\delta_{R F}^{4}\left(\delta_{T}^{2}+\delta_{R}^{2}\right)\left[1-\exp \left(-\delta_{T}^{2}\right)\right]\left[1-\exp \left(-\delta_{R}^{2}\right)\right]}$

The optimal LO field for a Gaussian transmitter field and a circular aperture was determined by Frehlich [8] and produces essentially the same performance as the design proposed by Rye. The behavior of heterodyne efficiency for a CLR with the Rye design will be considered here. 


\section{Simulation of Performance}

One important aspect of CLR performance for diffuse and aerosol targets is the random nature of the backscattered field. The superposition of many random backscattered fields with random amplitude and phase produces a complex Gaussian distribution or "speckle field" for the total backscattered field [9]. The detection of such random signals in random noise has been considered by Shapiro et al. [10]. We will investigate the estimation of the heterodyne efficiency by generating computer simulations of the normalized heterodyne power $c$ and the normalized direct detection power $d$. Realization of the fields on the detector will also be calculated to provide insight.

The backscattered field at the target can be represented as

$e_{S}(\overrightarrow{\mathbf{p}}, R)=U(\overrightarrow{\mathbf{p}}) e_{T}(\overrightarrow{\mathbf{p}}, R)$

where $U(\overrightarrow{\mathbf{p}})$ is a random complex amplitude with the property

$\left\langle U\left(\overrightarrow{\mathbf{p}}_{1}\right) U^{*}\left(\overrightarrow{\mathbf{p}}_{2}\right)\right\rangle=\lambda^{2} \delta\left(\overrightarrow{\mathbf{p}}_{\mathbf{1}}-\overrightarrow{\mathbf{p}}_{2}\right)$

where $\delta(\overrightarrow{\mathbf{p}})\left[\mathrm{m}^{-2}\right]$ is the two-dimensional delta function. The backscattered field at the receiver plane $e_{S}(\overrightarrow{\mathbf{v}}, 0)$ is given by

$e_{S}(\overrightarrow{\mathbf{v}}, 0)=\int_{-\infty}^{\infty} e_{S}(\overrightarrow{\mathbf{p}}, R) G(\overrightarrow{\mathbf{p}} ; \overrightarrow{\mathbf{v}}, R) d \overrightarrow{\mathbf{p}}$

The normalized direct detection power is then

$d=\frac{R^{2}}{A_{R} T_{T}} \int_{-\infty}^{\infty}|W(\overrightarrow{\mathrm{v}})|^{2}\left|e_{S}(\overrightarrow{\mathrm{v}}, 0)\right|^{2} d \overrightarrow{\mathrm{v}}$

and the normalized coherent detection power is

$c=\frac{R^{2}}{A_{R} T_{T}}\left|\int_{-\infty}^{\infty} e_{S}(\overrightarrow{\mathbf{v}}, 0) e_{B P L O}^{*}(\overrightarrow{\mathbf{v}}, 0) d \overrightarrow{\mathbf{v}}\right|^{2}$

The effects of angle misalignment $\Delta \theta$ are calculated by multiplying the BPLO field with no misalignment by the phase term [4] $\exp \left(i k \Delta \theta v_{x}\right)$ where $v_{x}[m]$ is the $\mathbf{x}$-coordinate of the vector $\overrightarrow{\mathbf{v}}$. The random fields are calculated using the Fast Fourier Transform (FFT) algorithm to propagate the fields. The random amplitudes $U(\overrightarrow{\mathbf{p}})$ are generated using the Box-Muller algorithm.

A typical receiver employs optical elements that pass all the backscattered field. Then, for ideal optical elements, the field on the detector surface is

$e_{S}(\overrightarrow{\mathbf{w}}, F)=\frac{k}{2 \pi i F} \int_{-\infty}^{\infty} W(\overrightarrow{\mathbf{v}}) e_{S}(\overrightarrow{\mathbf{v}}, 0) \exp \left(-\frac{i k}{F} \overrightarrow{\mathbf{v}} \cdot \overrightarrow{\mathbf{w}}\right) d \overrightarrow{\mathbf{v}}$

where $F[m]$ is the effective focal distance of the optical system. The fields on the detector surface are particulary intuitive since the heterodyne efficiency is unity when the backscattered field is proportional to the LO field. This is also true for the receiver-plane representation. 


\section{Results}

The simulation results for the far-field optimal design of Rye and the corresponding collimated near-field case with $\delta_{R F}=4$ will be presented. The statistical variability of the coherent detection power and the direct detection power from a simulation of 10,000 shots for the perfectly aligned CLR for the far-field case is shown in Fig. 1 and for the near-field case in Fig. 2. If the heterodyne efficiency had been unity, all the points would lie on a straight line with a slope of unity. The far-field case has more scatter than the near field case. This is due to the increased spatial averaging of the backscattered irradiance by the telescope aperture in the near-field case.

Typical realizations of the backscattered field on the detector surface for a perfectly aligned CLR is shown in Fig. 3 for the far-field and near-field cases. The dimensions of the central lobe is approximately the diffraction limit $\lambda F / a$ of the circular aperture. The poor match between the backscattered field and LO field for the typical far-field case is the reason the heterodyne efficiency is 0.46096 for the perfectly aligned far-field case. More than half the coherent power is lost due to the random mismatch between the backscattered and LO fields. The near-field case is characterized by a larger distribution of the field over the detector surface with fluctuations on the order of the diffraction limited scale but with reduced amplitude which results in a heterodyne efficiency of 0.021832 . The ratio of the heterodyne efficiency to the approximate near-field heterodyne efficiency Eq. (33) is 1.05554. The large number of "speckles" or regions of coherent field produces smaller variability of the direct detection signal (see Fig. 2). Also, for typical CLR designs, the detector would be smaller than the spatial extent of the field and the direct detection power would be reduced. Measurements of heterodyne efficiency in the near-field regime may contain a bias due to the finite extent of the detector. A rare case of high heterodyne efficiency and very low heterodyne efficiency is shown in Fig. 4 for the far-field case.

The sensitivity of heterodyne efficiency to the misalignment angle $\Delta \theta$ of the transmitter and BPLO axis $[1,6]$ is shown in Fig. 5 for the far-field and near-field cases. The theoretical heterodyne efficiency depends on the single parameter $\Delta q=k a \Delta \theta=\pi D \Delta \theta / \lambda$. The simulation results are in excellent agreement with the theory for both the near-field and far-field case. (The estimation error for each simulation data point is $1 \%$, consistent with exponential statistics for the coherent power.) The heterodyne efficiency was estimated by the ratio of the average of $c$ to the average of $d$ using the 10,000 shots of simulated data in Figs. 1 and 2 and various angle misalignment. In the laboratory one would measure the heterodyne efficiency in this way using a calibration target with a uniform backscatter coefficient $\rho$. The statistical accuracy of the estimate for heterodyne efficiency is determined by the number of shots averaged and the statistical distribution of the two estimates for $\langle c\rangle=\eta_{H}$ and $<d>=1$. The Probability Density Function (PDF) of the direct detection power $d$ and the Gamma Distribution function $g(d ; \alpha, \beta)$ proposed by Goodman [9] are shown in Fig. 6. The Gamma distribution function is given by

$g(d ; \alpha, \beta)=\frac{\alpha^{\beta}}{\Gamma(\beta)} d^{\beta-1} \exp (-\beta d)$

and the parameters of the Gamma Distribution are $\alpha=\beta=1 / \sigma_{d}^{2}$ where $\sigma_{d}^{2}$ is the variance of $d$. For the far-field case $\sigma_{d}=0.531$. The same comparison for the near-field case is shown in 
Fig. 7. Here, $\sigma_{d}=0.1526$. In both cases the simulation results are in reasonable agreement with the Gamma distribution.

The PDF of the normalized coherent detection power for the far-field case is shown in Fig. 8 for perfect alignment along with the theoretical exponential distribution. Because the backscattered field is the superposition of many, randomly phased components, the coherent power should have an Exponential PDF. This was verified by the simulations for all cases.

The estimate of heterodyne efficiency $\hat{\eta}_{H}$ with a fixed calibration target using the average of $J$ shots is given by

$\hat{\eta}_{H}=\frac{\sum_{k=1}^{J} c_{k}}{\sum_{k=1}^{J} d_{k}}=\frac{N}{M}$

where $c_{k}$ and $d_{k}$ are the coherent power and direct detection power for shot $k$. The numerator $N$ has a Gamma Distribution $g(N ; 1 /\langle c\rangle, J)$ and the denominator $M$ also has a Gamma Distribution $g\left(M ; 1 / \sigma_{d}^{2}, J / \sigma_{d}^{2}\right)$. For large $J$, the statistical accuracy of the estimate $\hat{\eta}_{H}$ is approximately

$$
\frac{\Delta \hat{\eta}_{H}}{\hat{\eta}_{H}}=\frac{\Delta N}{N}+\frac{\Delta M}{M}=\frac{1+\sigma_{d}}{\sqrt{J}}
$$

The estimation of heterodyne efficiency for satellite based lidars is a promising technique to verify and maintain beam alignment, but is more difficult since there is no calibration target. The surface return is the most promising signal since it may be large enough to have a detectable direct detection signal but there is added variability from shot to shot due to the random backscatter coefficient of the earths surface and the random atmospheric attenuation. This added variability produces larger scatter in the estimate of the coherent and direct-detection signal power. However, for estimation of heterodyne efficiency, this variability can be removed by defining a biased estimate of heterodyne efficiency for each shot as

$b=c / d$

provided the direct detection signal can be estimated accurately for every shot. The average of this biased estimate and it's standard deviation as a function of misalignment angle $\Delta \theta$ is shown in Fig. 9 along with the true heterodyne efficiency (see Fig. 5). The bias of the average of $b$ is small and therefore provides a useful absolute measure of beam alignment. The statistical reliability of this biased estimate depends on the PDF of $b$, which is shown in Fig. 10 for perfect alignment. Also shown is the PDF of the average of 10 estimates of $b$ and a Gaussian PDF with the correct mean value and variance given by the variance of $b$ divided by the number of shots averaged. The same results for a misalignment of $\Delta q=k a \Delta \theta=\pi D \Delta \theta / \lambda=2$ which corresponds to $2.08 \mathrm{~dB}$ loss in SNR are shown in Fig. 11. The Gaussian approximation for the PDF of the average of 10 estimates of $b$ is a good approximation. However, an accurate estimate of the biased heterodyne efficiency requires an average of many shots. The PDF of a 40 shot average of $b$ is shown in Fig. 12 for perfect alignment and misalignment of $\pi D \Delta \theta / \lambda=2(2.08 \mathrm{~dB}$ loss in SNR) and $\pi D \Delta \theta / \lambda=3(4.76$ $\mathrm{dB}$ loss in SNR), using the Gaussian approximation for the PDF's. The CLR misalignment can be reliably determined with 40 surface returns. 


\section{Detector Calibration}

A real doppler lidar system does not measure the signal currents directly. Therefore, detector calibration is required to extract the required signal currents from the detector signal. The heterodyne efficiency can be estimated using both Continuous Wave (CW) or pulsed mode of operation. For $C W$ operation, two signal currents $I_{S}(t)$ and $I_{D C}$ are DC signals which are given in terms of the detector voltage $V$ by

$I(V)=G_{D} h(V)$

where $I(V)$ is the photodetector response function $G_{D}[A / V]$ is the constant for a linear response, and $h(V)[V]$ is the nonlinearity function or deviation from linearity. We assume that the photodetector is ideal in the limit of small voltage, i.e., $h(V)=V$. For pulsed operation, the frequency response of the detector may be required to estimate the direct detection signal $I_{S}(t)$.

If the magnitude of the IF signal current $i_{S}(t)$ is small enough and if the magnitude of the IF voltage signal $v_{S}(t)[V]$ does not change with the IF frequency $\nu[H z]$ then

$i_{S}(t)=G_{D} f(V) v_{S}(t)$

where

$f(V)=\frac{1}{G_{D}} \frac{d I(V)}{d V}=\frac{d h(V)}{d V}$

is proportional to the derivative of the photodetector response function and $f(0)=1$. A measurement of heterodyne efficiency requires a measurement of $h(V)$ and $f(V)$, which can be performed by the method proposed by Frehlich [11]. (Note that Ref. [11] considered the calibration of integrated irradiance over the detector surface which is proportional to the detector current). The operating point $\left(I_{0}, V_{0}\right)$ determines the relationship between detector voltages and the ideal signal currents. The direct detection currents are given by

$I_{D C}=G_{D} h\left(V_{0}\right)$

and

$$
I_{S}(t)=G_{D} f\left(V_{0}\right) V_{S}(t)
$$

where $V_{S}(t)[V]$ is the increase in the DC detector voltage due to the backscattered field collected by the detector.

The effects of the detector electronics and detector quantum efficiency as a function of IF frequency are described by the normalized frequency response function

$H(\nu)=\frac{v_{S}(\nu)}{v_{S}(\nu \rightarrow 0)}$.

Then

$i_{S}(t)=G_{D} f\left(V_{0}\right) v_{S}(t) / H(\nu)$ 
and the heterodyne efficiency is given by [see Eq. (4)]

$\eta_{H}(t)=\frac{f\left(V_{0}\right)\left\langle v_{S}^{2}(t)\right\rangle}{2 h\left(V_{0}\right)\left\langle V_{S}\right\rangle H^{2}(\nu)}$

which is expressed in terms of the calibration functions $f\left(V_{0}\right), h\left(V_{0}\right), H(\nu)$, and the detector voltages $v_{S}(t)$ and $V_{S}(t)$. Note that the ideal detector gain $G_{D}$ is not required. The sensitivity of the calibration functions to the detector operating point $\left(I_{0}, V_{0}\right)$ should be investigated.

\section{Summary}

The estimation of heterodyne efficiency is an excellent measure of CLR performance since it is proportional to SNR but independent of the transmitter power, the target backscatter coefficient, the atmospheric attenuation, and the detector quantum efficiency and gain. The heterodyne efficiency is an absolute measure of the alignment of the backscattered and LO field on the detector surface. The estimation of heterodyne efficiency is a statistical problem because the detector signals are random. An estimation of the heterodyne efficiency requires an accurate average of the coherent detection power, the direct detection power, and the LO current. When using a calibration target, an unbiased estimate of the heterodyne efficiency can be obtained by an ensemble average of the coherent and direct detection power. For a lidar in space, the ratio of the coherent detection power to the direct detection power for each ground return is a convenient biased estimate of heterodyne efficiency. The average of this biased estimate produces a sensitive measure of CLR alignment and removes the statistical variability of the random fluctuations of the signals due to the random backscatter coefficient of the earth surface and the random atmospheric attenuation. Using 40 shots produces an effective measure of system performance and beam alignment [see Fig. 12]. All the results presented here assume a large detector that collects all the LO power and all the backscattered power.

The calibration of real detectors requires two measurements: the low frequency response function $h(V)$ (which is obtained [11] from a measurement of $f(V)$, the derivative of $h(V)$ ) and the frequency response $H(\nu)$.

\section{Acknowledgments}

The author acknowledges useful discussions with M. J. Kavaya, B. J. Rye, and G. Spiers. This work was supported by the National Science Foundation and the National Aeronautics and Space Administration Marshall Space Flight Center under Research Grant NAG8-253 (Michael J. Kavaya, Technical Officer). 


\section{References}

1. R. G. Frehlich and M. J. Kavaya, "Coherent laser radar performance for general atmospheric refractive turbulence," Appl. Opt. 30, 5325-5352 (1991).

2. Y. Zhao, M. J. Post, and R. M. Hardesty, "Receiving efficiency of monostatic pulsed coherent lidars. 1: Theory," Appl. Opt. 29, 4111-4119 (1990).

3. G. O. Olaofe, "Diffraction by gaussian apertures," J. Opt. Soc. Am. 60, 1654-1657 (1970).

4. R. G. Frehlich, "Effects of refractive turbulence on coherent laser radar," Appl. Opt. 32, 2122-2139, (1993)

5. J. Y. Wang, "Optimum truncation of a lidar transmitted beam," Appl. Opt. 27, 44704474 (1988).

6. B. J. Rye, "Primary aberration contribution to incoherent backscatter heterodyne lidar returns," Appl. Opt. 21, 839-844 (1982).

7. B. J. Rye and R. G. Frehlich, "Optimal truncation of Gaussian beams for coherent lidar using incoherent backscatter," Appl. Opt. 31, 2891-2899 (1992).

8. R. . G. Frehlich, "Optimal design of monostatic coherent laser radar with a circular aperture", Appl. Opt. 32, 4569-4577 (1993).

9. J. W. Goodman, "Some effects of target-induced scintillation on optical radar performance," Proc. IEEE, 53, 1688-1700 (1965)

10. Shapiro, J.H., B.A. Capron, and R.C. Harney, "Imaging and target detection with a heterodyne-reception optical radar" Appl. Opt. 20, 3292-3313 (1981).

11. R. G. Frehlich, "Estimation of the non-linearity of a photodetector," Appl. Opt. 31, 5926-5929, (1992). 


\section{Figure 1}

Fig. 1. Normalized coherent detection power $c$ versus the normalized direct detection power $d$ for a perfectly aligned CLR and 10,000 shots with the target in the far field.

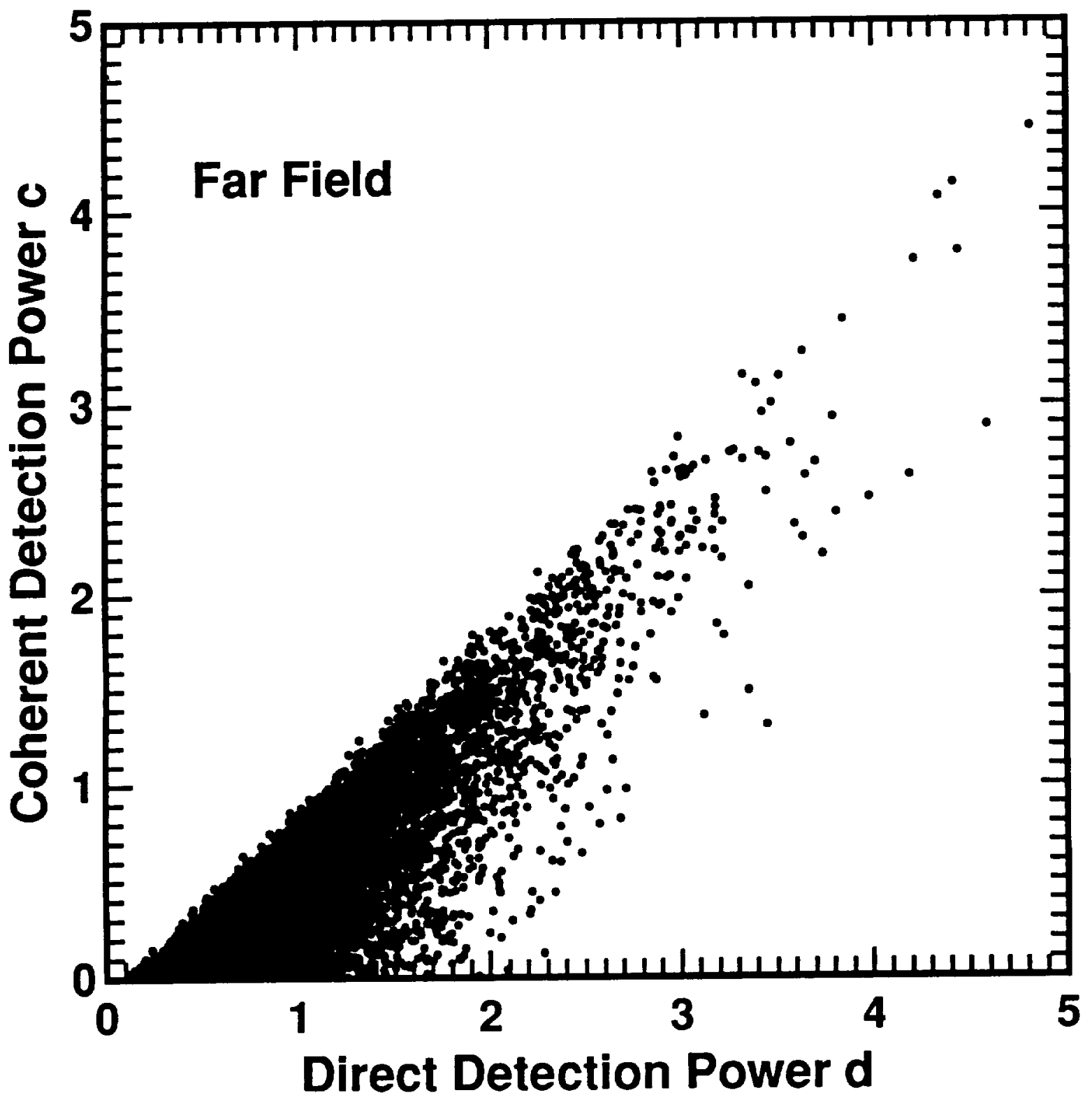


Figure 2

Fig. 2. Normalized coherent detection power $c$ versus the normalized direct detection power $d$ for a perfectly aligned CLR and 10,000 shots with the target in the near field $\left(\delta_{R F}=a / R_{F}=4\right)$.

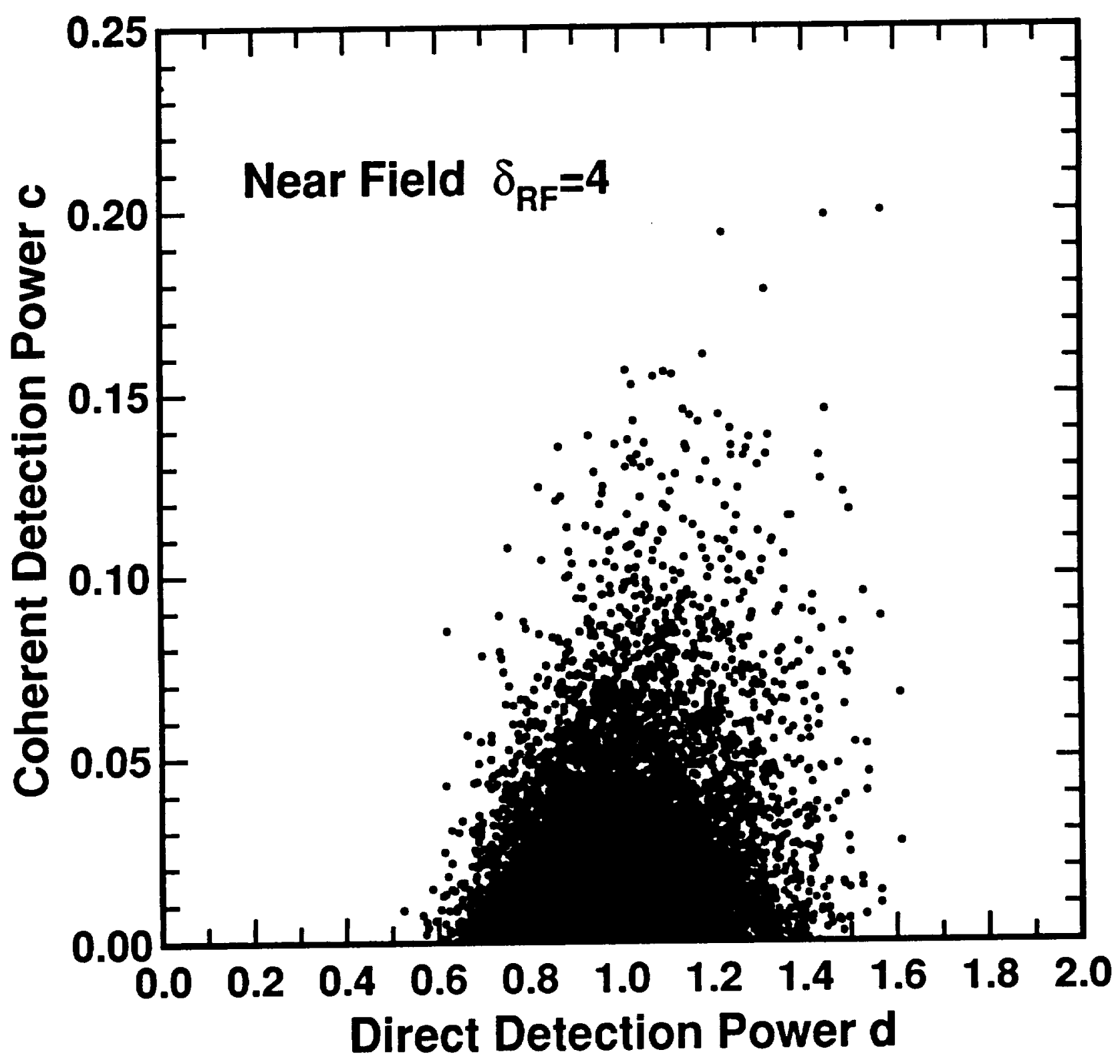


Fig. 3. Simulation of the backscattered field on the detector surface in the $\mathrm{x}$ direction $e_{S}\left(w_{x}, F\right)$ and the $y$ direction $e_{S}\left(w_{y}, F\right)$ for typical far field $\left(\eta_{H}=0.460, d=1.015, c=0.467\right)$ and near field with $\delta_{R F}=a / R_{F}=4\left(\eta_{H}=0.0785, d=0.969, c=0.0760\right)$ cases. The real part of the complex field is ( - ) and the imaginary part is $(\cdots)$. The optimal LO field $e_{L O}(w, F)$ is also shown.

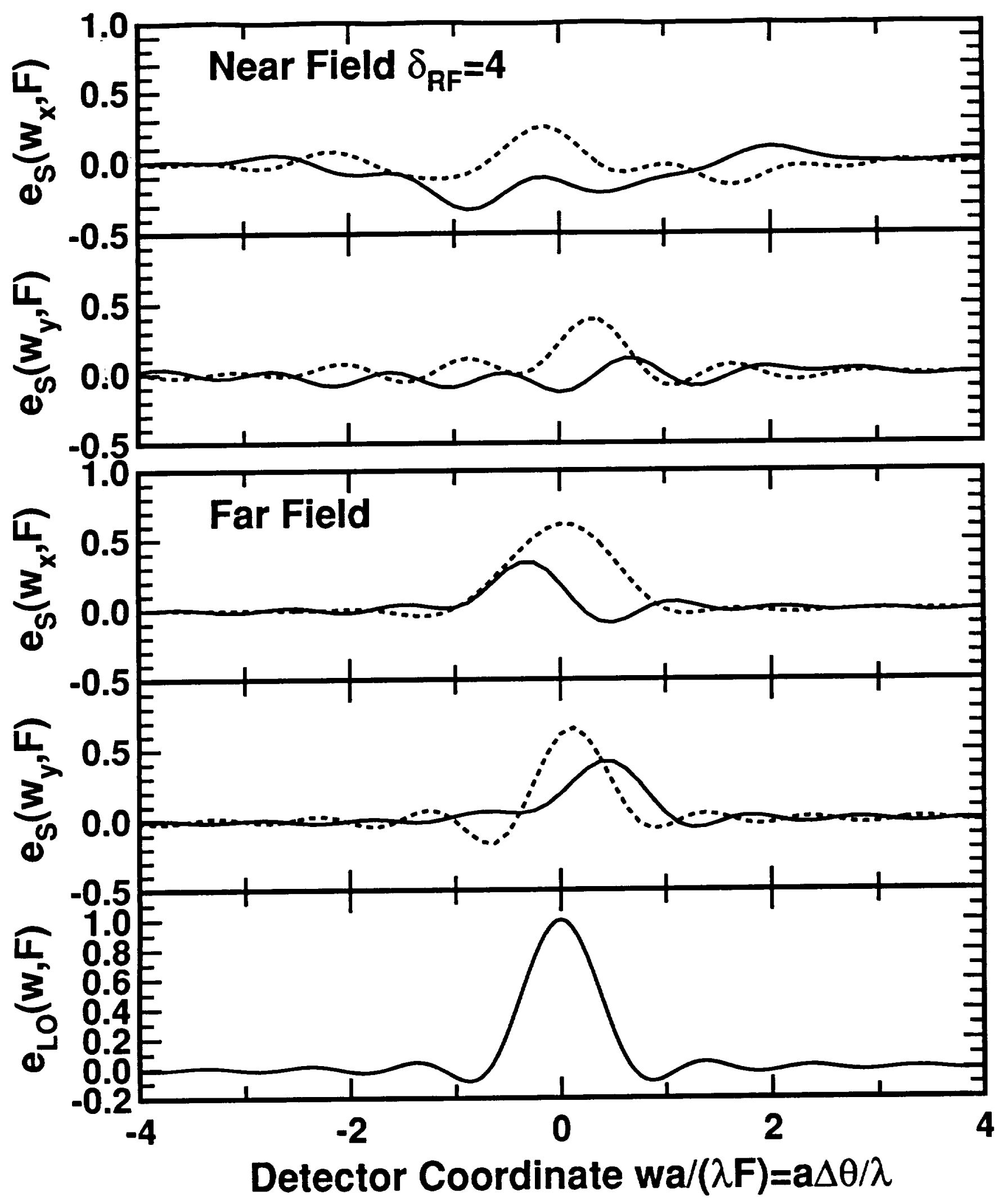


Fig. 4. Simulation of the backscattered field on the detector surface in the $\mathrm{x}$ direction $e_{S}\left(w_{x}, F\right)$ and the $y$ direction $e_{S}\left(w_{y}, F\right)$ for far field and rare cases of excellent heterodyne efficiency $\left(\eta_{H}=0.9465, d=3.325, c=3.147\right)$ and poor heterodyne efficiency $\left(\eta_{H}=0.000344, d=0.830\right.$, $c=0.000286)$. The real part of the complex field is $(-)$ and the imaginary part is $(\cdots)$. The optimal LO-field $e_{L O}(w, F)$ is also shown.

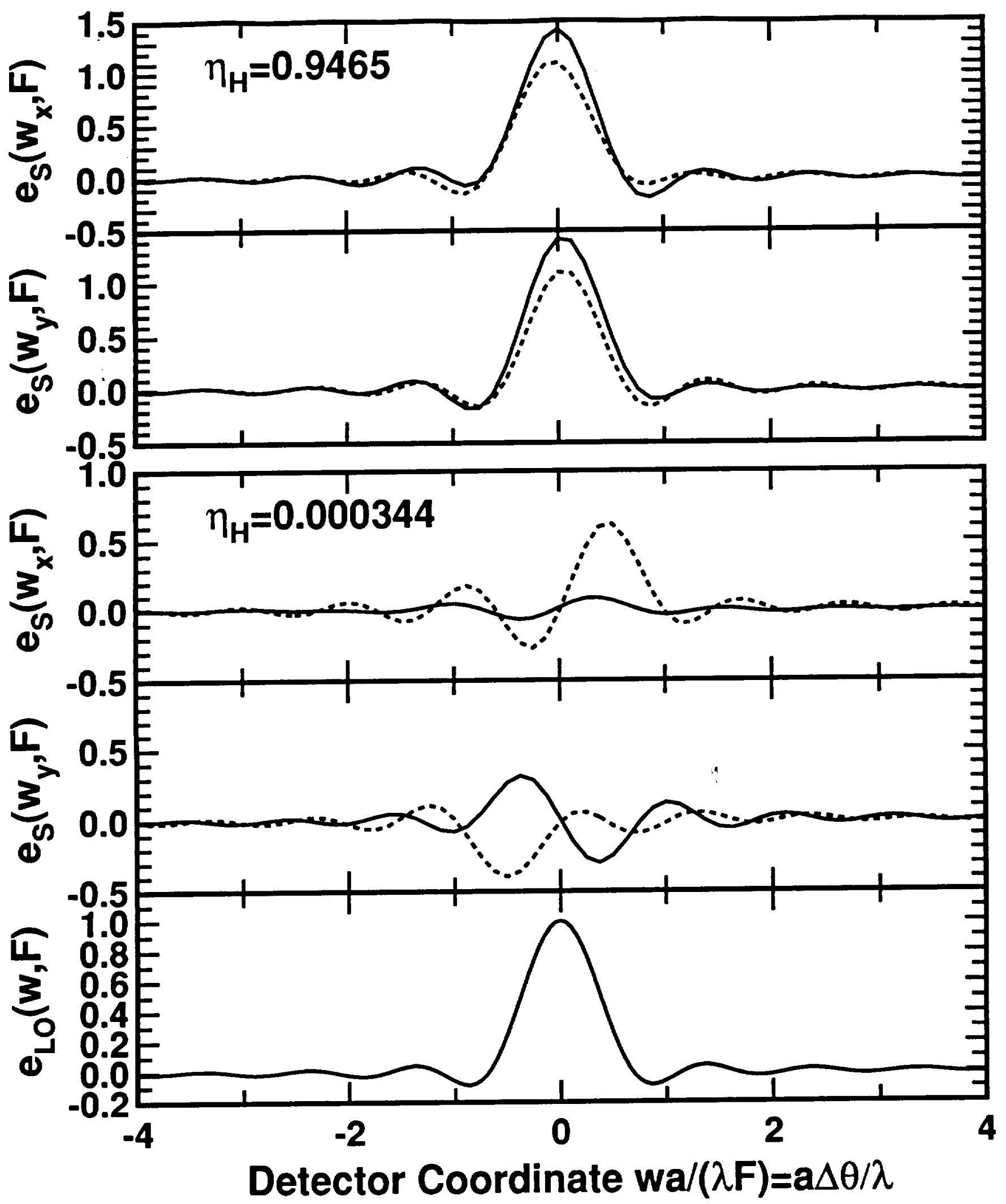




\section{Figure 5}

Fig. 5. Heterodyne efficiency $\eta_{H}$ as a function of misalignment $\pi D \Delta \theta / \lambda$ with the results of the 10,000 shot simulation of Fig. 1 for the far-field $(\bullet)$ and near-field $(0)$ cases with $\delta_{R F}=a / R_{F}=4$. The theoretical calculations Eq. (31) are (-). The best-fit Gaussian function $0.46096 \exp \left[-(\pi D \Delta \theta / 2.8363 \lambda)^{2}\right]$ is $(\cdots)$ and the best fit power-law model $0.9157(\pi D \Delta \theta / \lambda)^{-3.0415}$ is $(\cdots)$.

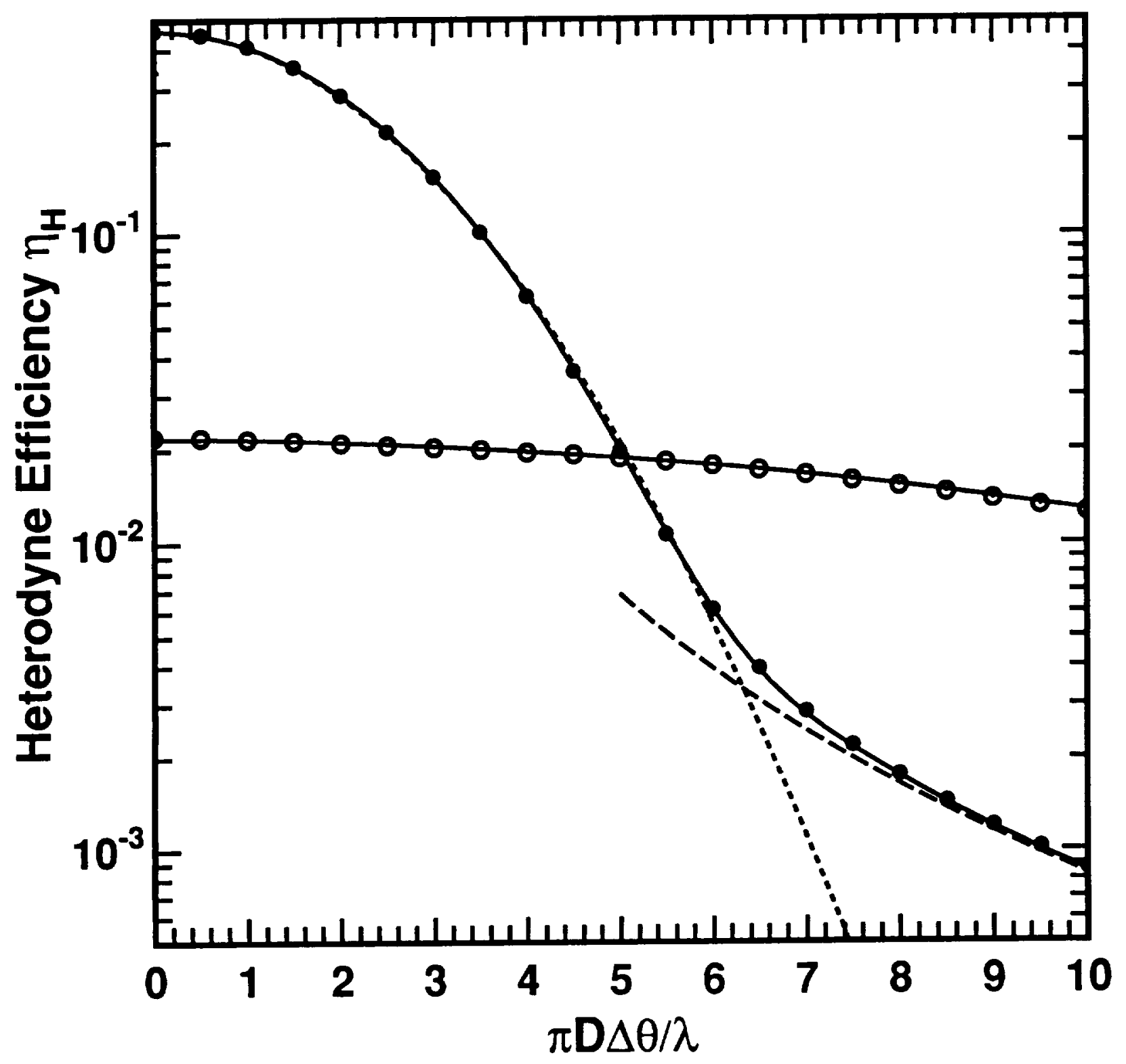




\section{Figure 6}

Fig. 6. PDF of direct detection power $d$ from simulation of Fig. 1 and the theoretical Gamma distribution (-) for a perfectly aligned CLR in the far-field.

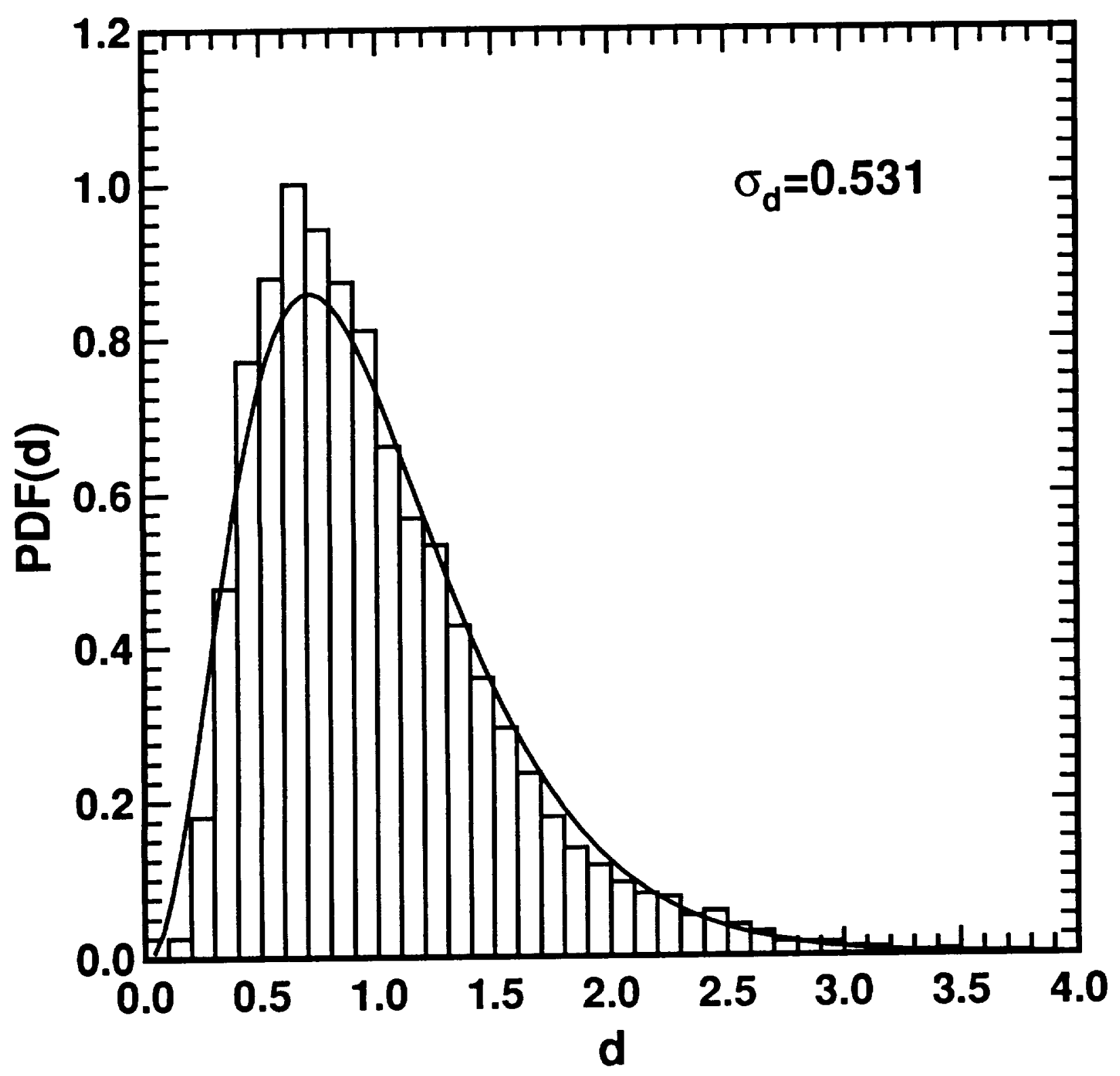




\section{Figure 7}

Fig. 7. PDF of direct detection power $d$ using the average of 10 shots from the simulation of Fig. 1 and the theoretical Gamma distribution (-) for a perfectly aligned CLR in the far-field.

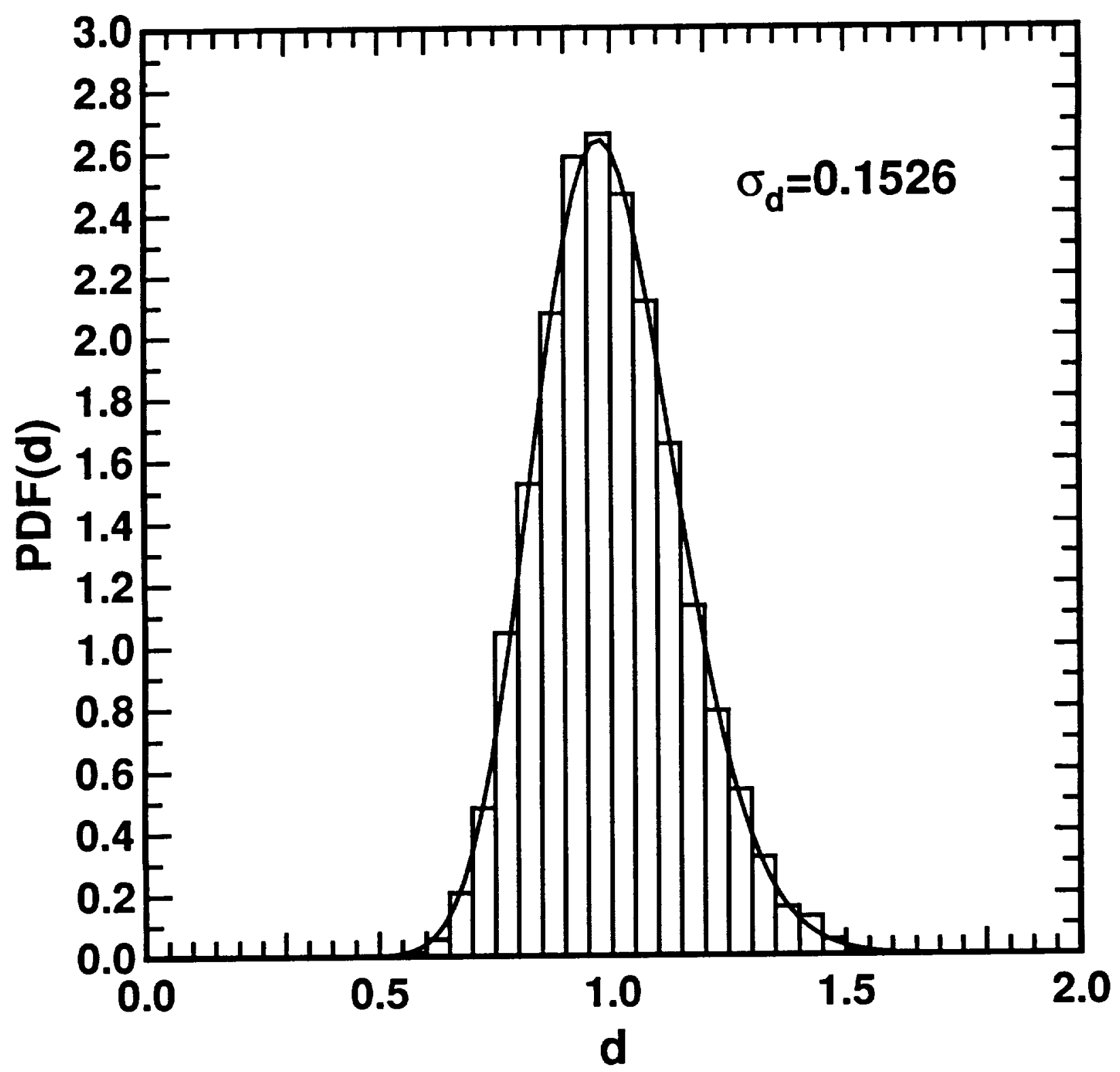


Figure 8

Fig. 8. PDF of coherent detection power $c$ from simulation of Fig. 1 and the theoretical Exponential distribution (-) for a perfectly aligned CLR in the far-field.

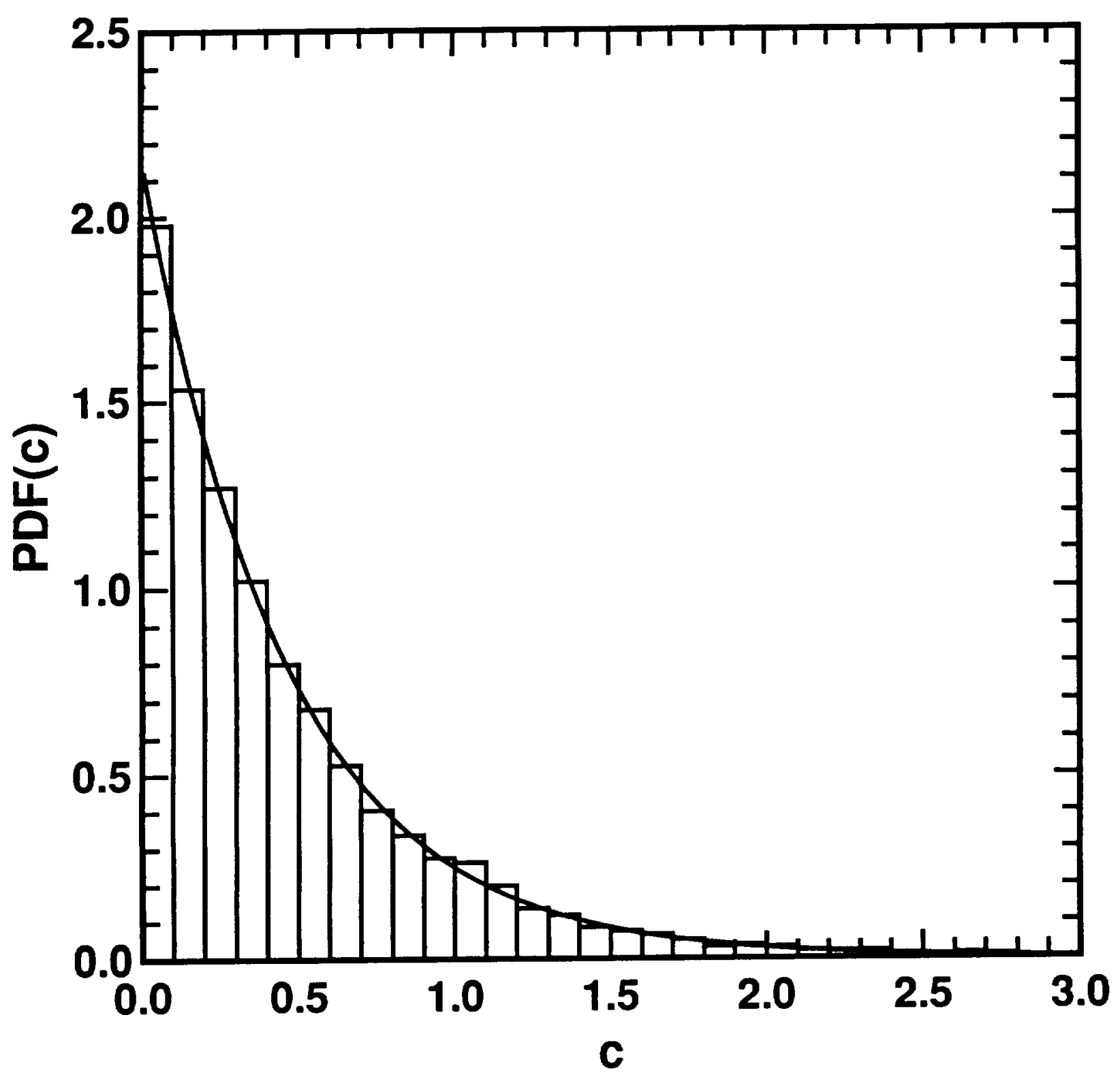




\section{Figure 9}

Fig. 9. Biased estimates of heterodyne efficiency $b=c / d$ as a function of misalignment $\pi D \Delta \theta / \lambda$.

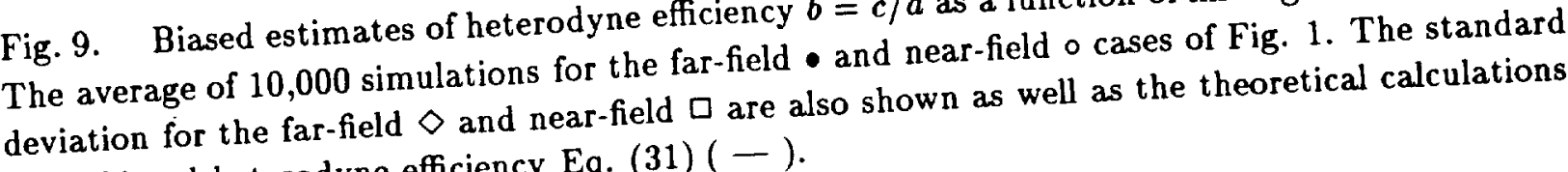
for unbiased heterodyne efficiency Eq. (31) ( - ).

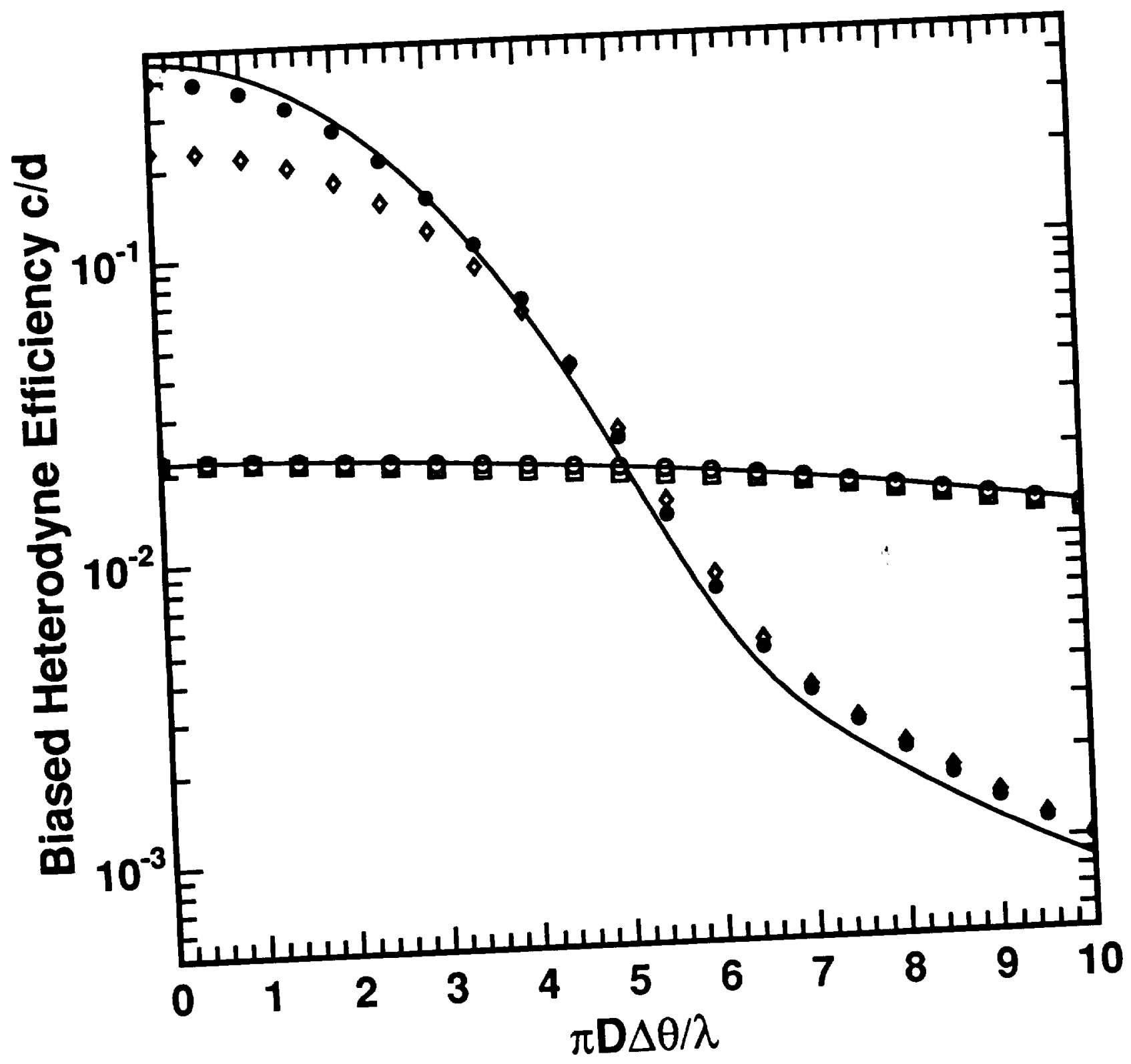




\section{Figure 10}

Fig. 10. PDF of the biased estimate of heterodyne efficiency $b=c / d$ for perfect alignment in the far-field for 1 shot and the average of 10 shots. The Gaussian PDF ( - ) with the estimated mean $\langle b\rangle$ and standard deviation $\sigma_{b}$.

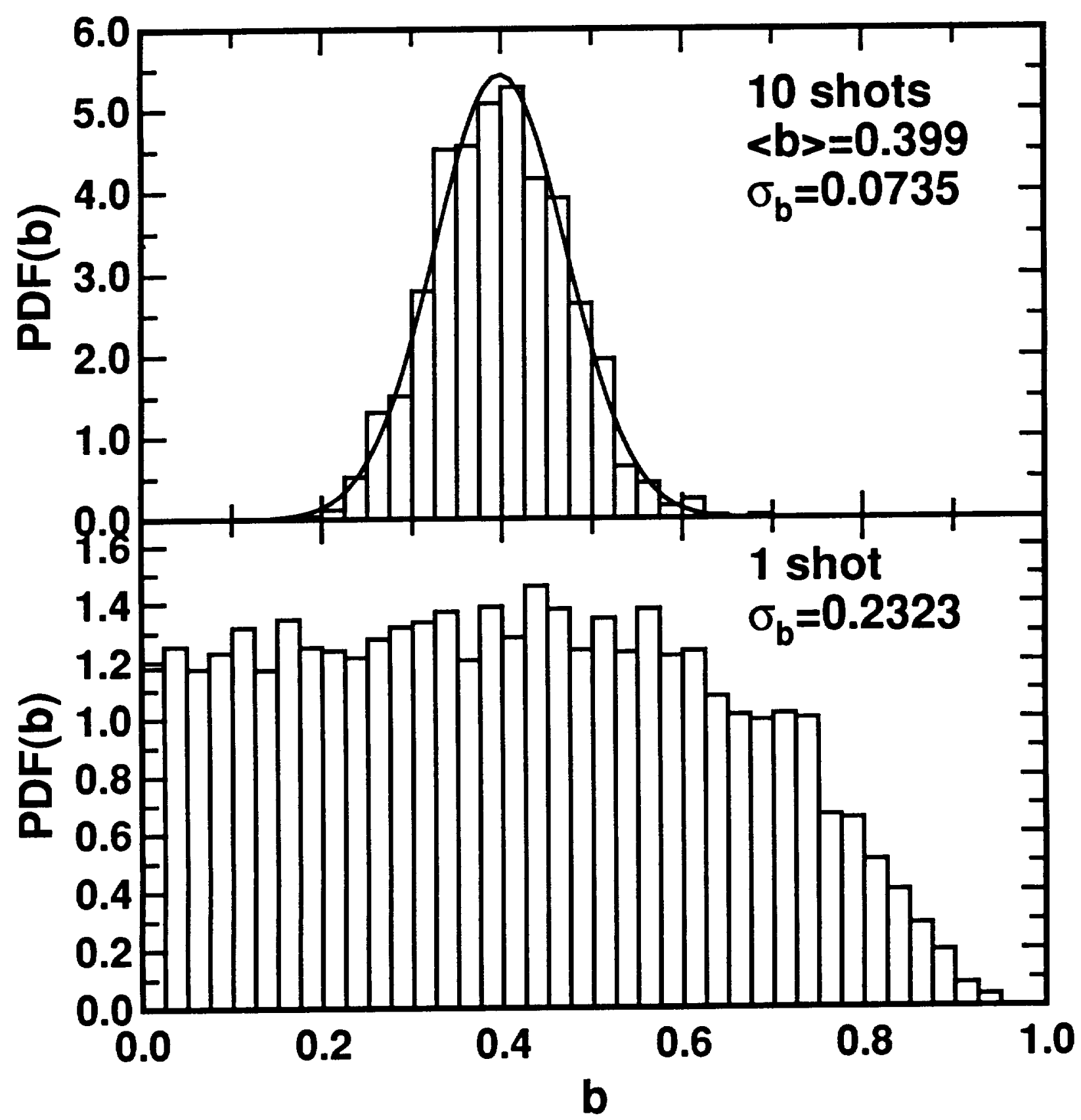




\section{Figure 11}

Fig. 11. PDF of the biased estimate of heterodyne efficiency $b=c / d$ for a misalignment of $\pi D \Delta \theta / \lambda=2$ in the far-field for 1 shot and the average of 10 shots. The Gaussian PDF ( - ) with the estimated mean $\langle b\rangle$ and standard deviation $\sigma_{b}$.

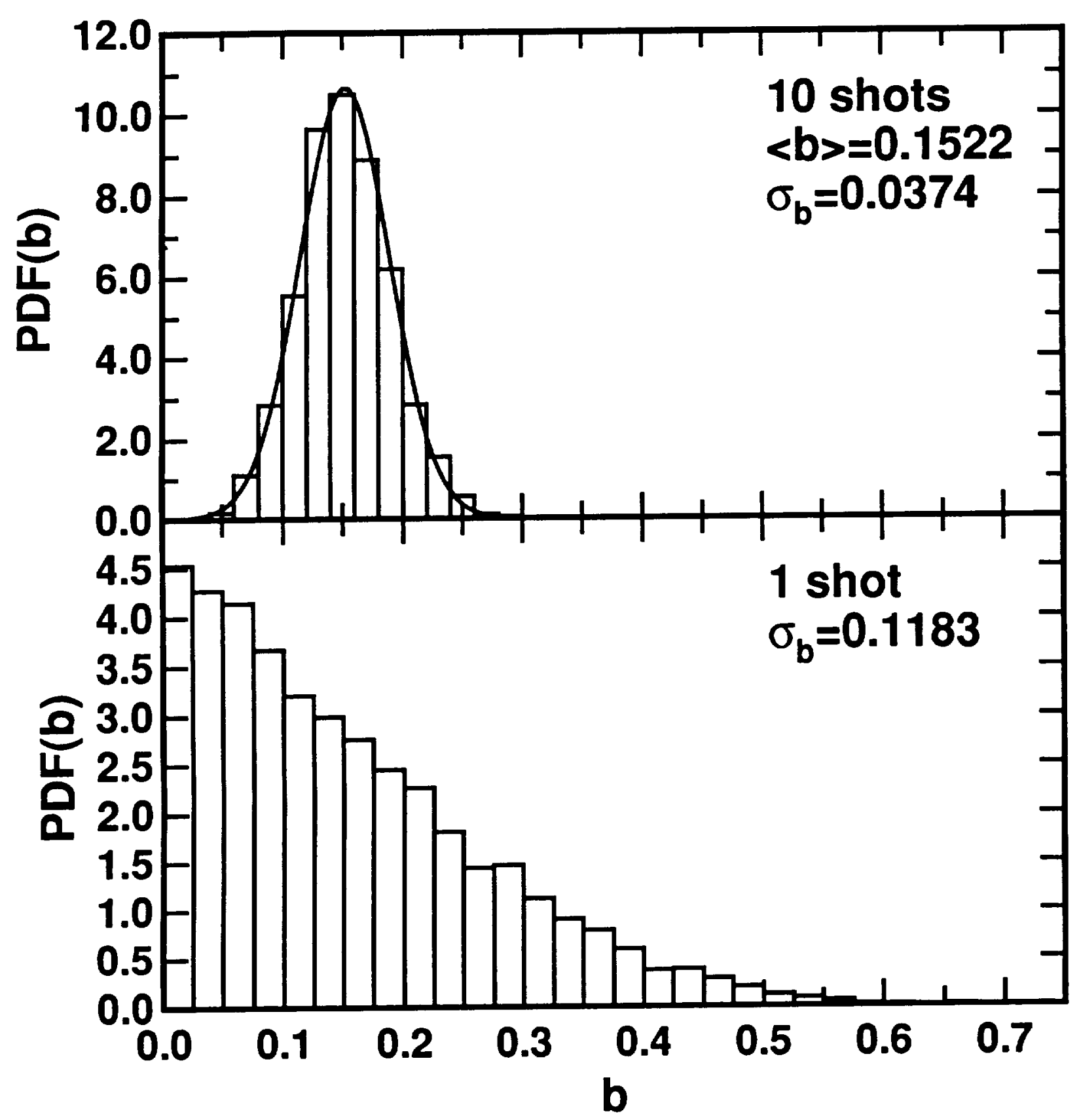


Figure 12

Fig. 12. PDF of the average of 40 estimates of $b$ for a misalignment of $\pi D \Delta \theta / \lambda=0(-), 2$ $(\cdots)$, and $3(---)$ assuming a Gaussian PDF.

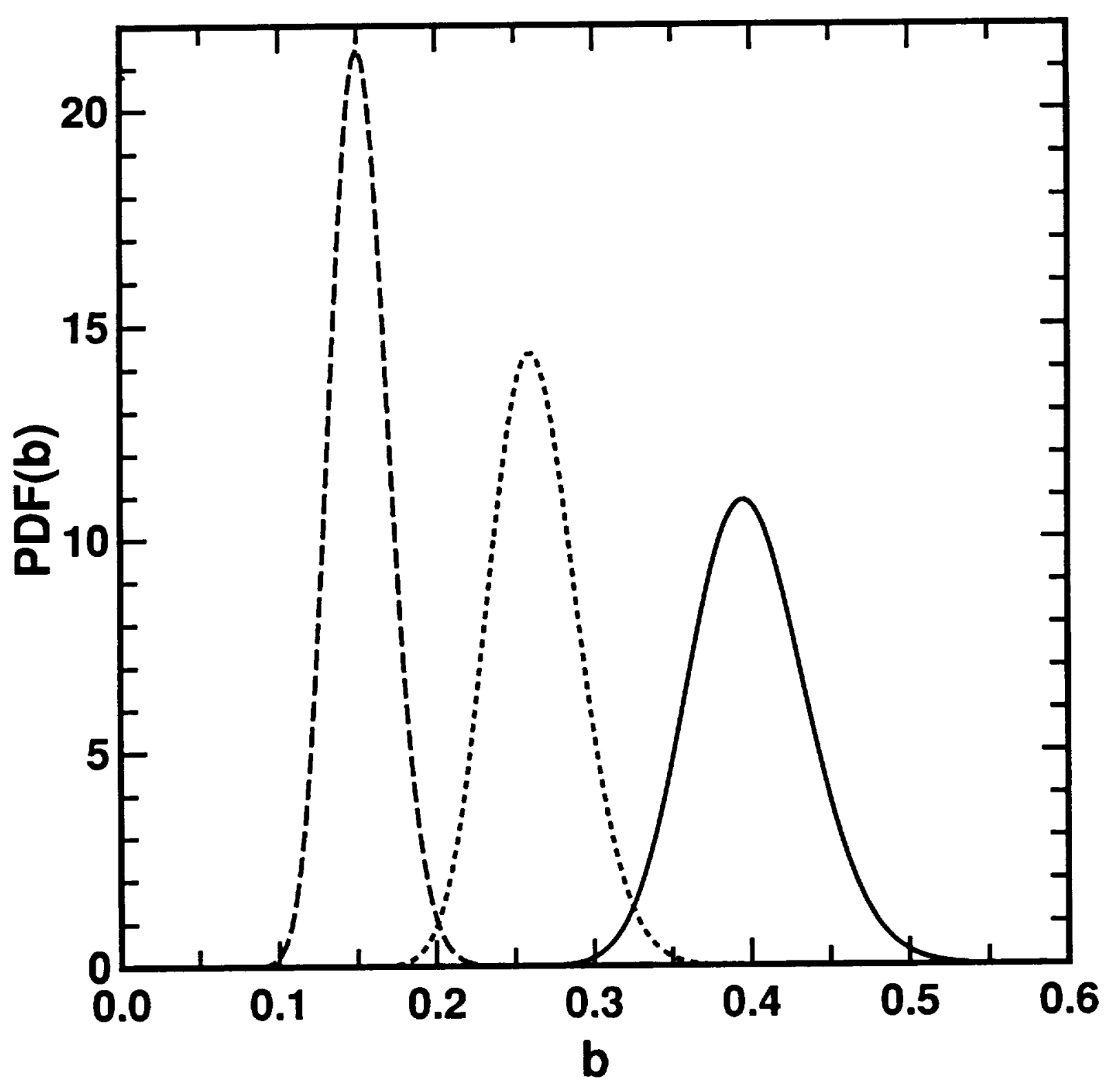

